\title{
A hazardous finding of a rare anomalous left main coronary artery in a patient with a secundum atrial septal defect
}

\author{
Michael Emery' \\ Waqas Ghumman' \\ Shawn Teague ${ }^{2}$ \\ Jo Mahenthiran' \\ 'Krannert Institute of Cardiology, \\ 2Department of Radiology, Indiana \\ University School of Medicine, \\ Indianapolis, Indiana, USA
}

Correspondence: Jo Mahenthiran Indiana University School of Medicine, Krannert Institute of Cardiology, I80 I N Senate Blvd, MPC2, Suite 4000, Indianapolis, IN 46202, USA

Tel + I 3179620539

Fax + I 3179620116

Email jmahenth@iupui.edu

\begin{abstract}
A 23-year-old male referred for evaluation of a "choking" sensation with exertion and a murmur. A transthoracic echocardiogram demonstrated right atrial and ventricular dilatation, right ventricular volume overload, and a large secundum atrial septal defect (ASD) with left to right shunt and a calculated pulmonary-to-systemic blood flow ratio $(Q p / Q s)$ estimated at 2.3 to 1. Cardiac catheterization also demonstrated evidence of the ASD with $Q p / Q s$ of 4.6 to 1 with a significant step-up in oxygen saturation at the right atrial level. Additionally, an anomalous left main coronary artery (ALMCA) origin from the anterior right coronary cusp was suspected. Using 64-slice multidetector computed tomography coronary angiography (CCTA) the left main coronary artery was seen to arise from the right coronary cusp then traverse between the pulmonary trunk and the proximal ascending aorta before bifurcating into the left anterior descending and circumflex arteries that followed their normal courses distally. Based on the high risk nature of associated sudden death from an anomalous left main coronary artery (ALMCA) coursing between the aorta and the pulmonary trunk, the patient underwent surgical re-implantation of the ALMCA to the left coronary cusp and repair of the ASD. This case highlights a rare finding of a hazardous ALMCA in a patient with a secundum ASD and the utility of CCTA in evaluating the course of coronary anomalies along with other cardiac pathology.
\end{abstract}

Keywords: atrial septal defect, anomalous coronary artery, congenital heart disease

We report a case of a 23-year-old Hispanic male who was referred for evaluation of a "choking" sensation with exertion and a murmur. The patient's past medical history was significant for hypertriglyceridemia for which he was on gemfibrozil. He described a sensation of choking with mild dyspnea during activities that would promptly resolve with rest. He denied any other associated symptoms such as chest discomfort, diaphoresis, syncope, lightheadedness, palpitations or nausea. These symptoms had been present for several months and led him to stop participating in strenuous activities.

On physical exam, pulse was 72 beats per minute and blood pressure 134/64 mmHg. His apical impulse was not clearly palpable, but there was a prominent right ventricular lift. Cardiac auscultation was notable for regular rate and rhythm and a narrowly split second heart sound with reduced respiratory variation. An II/VI early systolic ejection murmur; a soft, early decrescendo diastolic murmur along the left sternal border; and a prominent mid-diastolic murmur at the left lower sternal border were audible. Lungs were clear and the rest of his exam was unremarkable. 12-lead electrocardiogram showed normal sinus rhythm with Wenckebach type atrio-ventricular block, incomplete right bundle branch block and non-specific ST-T wave changes. 


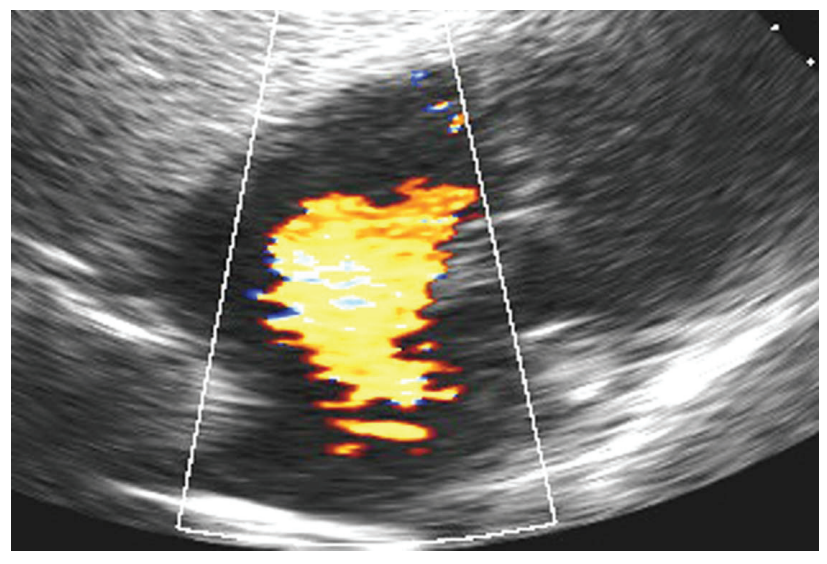

Figure I Transthoracic echocardiogram in the subcostal view with color Doppler flow imaging showing the large left to right shunt of a secundum atrial septal defect.

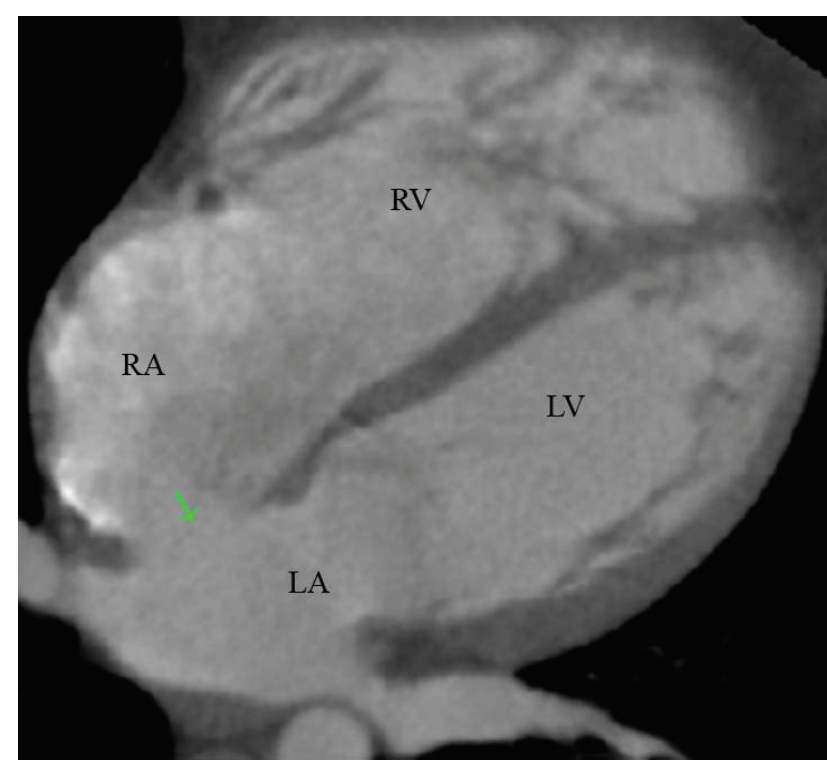

Figure 2 Cardiac computed tomography showing the large secundum atrial septal defect (green arrow) as well as the dilated right atrium (RA) and right ventricle (RV); LA (left atrium), LV (left ventricle).

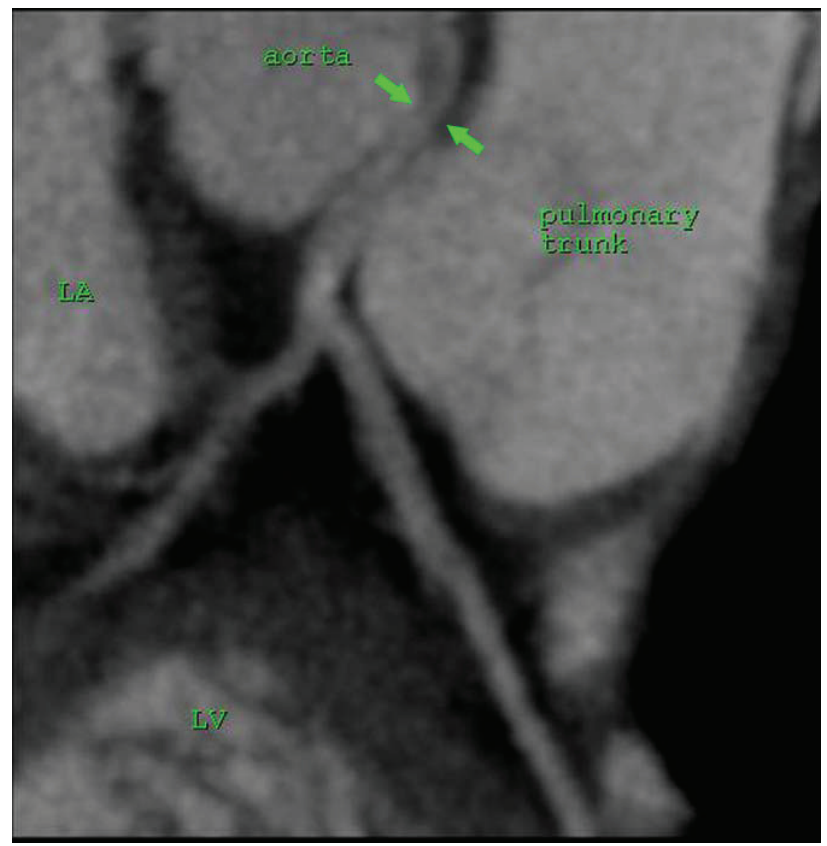

Figure 3 Curved multiplanar reformat of computed tomography coronary angiography demonstrating the anomalous left main coronary artery (green arrows) arising from the right coronary cusp then traversing between the aorta and the pulmonary trunk before bifurcating into the left anterior descending and circumflex arteries. LA (left atrium), LV (left ventricle).

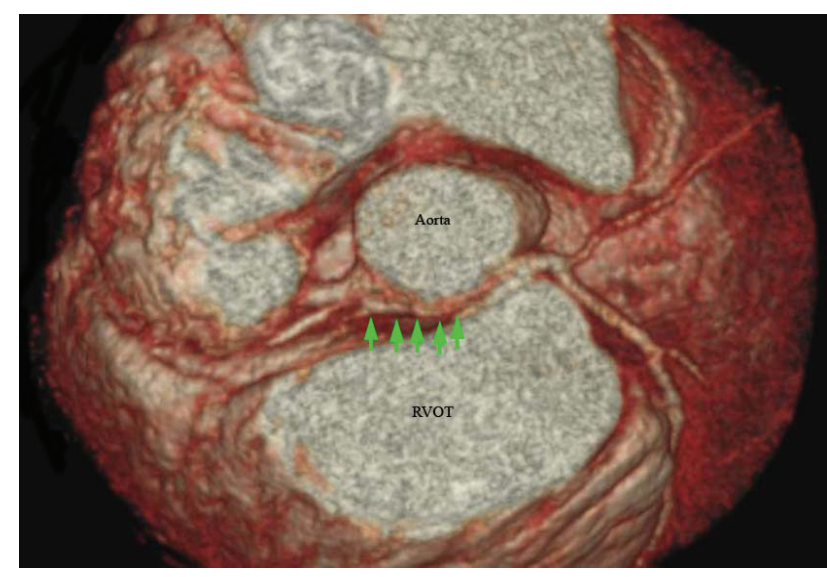

Figure 4 Three-dimensional reconstructed, volume rendered computed tomography coronary angiography showing the course of the anomalous left main coronary artery (green arrows) arising from the right coronary cusp then traversing between the aorta and right ventricular outflow tract (RVOT). 
A transthoracic echocardiogram obtained to further evaluate the murmur revealed normal left ventricular systolic function and hyperdynamic right ventricular systolic function with normal wall motion. Left ventricular and left atrial sizes were normal, but there was right ventricular and right atrial dilatation along with right ventricular volume overload. A large secundum atrial septal defect (ASD) with left to right shunt was evident (Figure 1) with a calculated pulmonary-to-systemic blood flow ratio $(Q p / Q s)$ estimated at 2.3 to 1 .

His symptoms of "choking" with exertion, however, were not typical presenting symptoms in an isolated ASD. As such, a right and left heart catheterization were performed to further evaluate his right sided heart pressures, shunt fraction and coronary anatomy. Pulmonary artery pressure was 29/9 $\mathrm{mmHg}$ (mean $16 \mathrm{mmHg}$ ) with a mean right atrial pressure of $6 \mathrm{mmHg}$. Mixed venous saturation was $73 \%$, right atrial saturation $85 \%$ and pulmonary artery saturation $96 \%$. $Q p / Q s$ was calculated to be 4.60 . Subsequent coronary angiography demonstrated a right dominant system with a normal appearing right coronary artery. The left main coronary artery was found to anomalously originate from the anterior right coronary cusp. The left main, left circumflex and left anterior descending artery appeared to be angiographically normal; however, we were not able to reliably determine its anatomic course in relation to the great vessels.

Because of the potential high-risk of sudden cardiac death in patients with an anomalous left main coronary artery (ALMCA), we felt it was prudent to completely define the route of the left main coronary artery in relation to the great vessels as an inter-arterial course between the aorta and pulmonary artery would warrant surgical intervention. As such, a 64-slice multidetector computed tomography coronary angiography (CTCA) was performed to further evaluate the coronary anatomy and ASD. The CTCA demonstrated a large ASD along with a dilated right ventricle and right atrium (Figure 2). $Q p / Q s$ was 2.3 by CTCA using left and right ventricular stroke volumes. The left main coronary artery was seen to arise from the right coronary cusp then traverse between the pulmonary trunk and the proximal ascending aorta before bifurcating into the left anterior descending and circumflex arteries, which then followed their normal courses distally (Figures 3 and 4). All vessels were patent with no critical coronary artery stenoses.

The risk of death from an ALMCA coursing between the aorta and the pulmonary trunk is difficult to evaluate as most cases are first recognized at autopsy and are rarely suspected or identified during life (Frescura et al 1998; Basso et al 2000). Most estimates of risk for sudden cardiac death have ranged from 36\%-59\% (Angelini et al 2002; Moustafa et al 2007). Basso and colleagues (2000) suggest that operation is probably mandatory for these patients. Given this high risk of sudden cardiac death, the patient underwent surgical re-implantation of the ALMCA to the left coronary cusp and repair of the ASD. He was discharged home after an uneventful hospital course, and on follow-up is doing well with complete resolution of his symptoms.

ASD is the second most common form of congenital heart disease found in adults (30\%) with the secundum type being the most common (75\%) (Wu and Child 2004). The incidence of ALMCA from the right coronary cusp is rare in the general population $(0.02 \%)$ and one of the less frequent types of anomalous coronaries (1.3\%) (Yamanaka and Hobbs 1990). Anomalous coronary arteries are more commonly associated with other congenital heart defects like tetralogy of Fallot, transposition of the great arteries, double outlet right ventricle, univentricular hearts, and truncus arteriosus (Vlodaver et al 1975). There have been case reports of other coronary anomalies in patients with an ASD such as an anomalous right coronary artery (Maki et al 2001) and anomalous left coronary artery from pulmonary artery (Turley et al 1995; Schneider et al 2006); however, to our knowledge this is the first reported case of an isolated secundum ASD and ALMCA with an inter-arterial course in the same patient. CTCA is a well recognized and appropriate modality for the evaluation of suspected anomalous coronary arteries (Hendel et al 2006) and has a high negative predictive value for excluding critical coronary artery stenoses (Achenbach 2007). It may also be a valuable tool in patients with ASDs for further evaluation of concomitant cardiac pathology, shunt calculation and coronary stenoses prior to referral for definitive therapy.

\section{Disclosure}

The authors report no conflicts of interest.

\section{References}

Achenbach S. 2007. Cardiac CT: State of the art for the detection of coronary arterial stenosis. J Cardiovasc Comput Tomogr, 1:3-20.

Angelini P, Velasco JA, Flamm S. 2002. Coronary Anomalies: Incidence, Pathophysiology, and Clinical Relevance. Circulation, 105:2449-54.

Basso C, Maron BJ, Corrado D, et al. 2000. Clinical profile of congenital coronary artery anomalies with origin from the wrong aortic sinus leading to sudden death in young competitive athletes. $J$ Am Coll Cardiol, 35:1493-1501.

Frescura C, Basso C, Thiene G, et al. 1998. Anomalous origin of coronary arteries and risk of sudden death: A study based on an autopsy population of congenital heart disease. Hum Pathol, 29:689-95. 
Hendel RC, Patel MR, Kramer CM, et al. 2006. ACCF/ACR/SCCT/SCMR/ ASNC/NASCI/SCAI/SIR 2006 Appropriateness Criteria for Cardiac Computed Tomography and Cardiac Magnetic Resonance Imaging: A Report of the American College of Cardiology Foundation Quality Strategic Directions Committee Appropriateness Criteria Working Group, American College of Radiology, Society of Cardiovascular Computed Tomography, Society for Cardiovascular Magnetic Resonance, American Society of Nuclear Cardiology, North American Society for Cardiac Imaging, Society for Cardiovascular Angiography and Interventions, and Society of Interventional Radiology. J Am Coll Cardiol, 48:1475-97.

Maki F, Ohtsuka T, Suzuki M, et al. 2001. Myocardial ischemia induced by anomalous aortic origin of the right coronary artery in a patient with atrial septal defect. Japan Heart J, 42:371-6.

Moustafa SE, Zehr K, Mookadam M, et al. 2007. Anomalous interarterial left coronary artery: An evidence based systematic overview. Int $J$ Cardiol, In Press, Aug 13.
Schneider T, Rickli H, Gliech V, et al. 2006. Bland-White-Garland syndrome and atrial septal defect - rare association and diagnostic challenge. Clin Res Cardiol, 95:295-300.

Turley K, Szarnicki RJ, Flachsbart KD, et al. 1995. Aortic implantation is possible in all cases of anomalous origin of the left coronary artery from the pulmonary artery. Ann Thorac Surg, 60:84-9.

Vlodaver Z, Neufeld HN, Edwards JE. 1975. Coronary Artery Variations in the Normal Heart and in Congenital Heart Disease. San Diego: Acad Pr.

Wu JC, Child JS. 2004. Common congenital heart disorders in adults. Curr Prob Cardiol, 29:641-700.

Yamanaka O, Hobbs RE. 1990. Coronary artery anomalies in 126,595 patients undergoing coronary arteriography. Cathet Cardiovasc Diagn, 21:28-40. 